\title{
The Kamin effect as a function of time of training and associative-nonassociative processes
}

\author{
JEFFREY A. SEYBERT, MARK A. WILSON, and ALAN L. ARCHER \\ University of Missouri, Kansas City, Missouri 66210
}

\begin{abstract}
In Experiment 1, for animals trained in the morning, Kamin effects (U-shaped retention functions) were obtained for groups receiving active avoidance training (AA) or noncontingent shocks in the avoidance apparatus (PNC), but not for animals receiving noncontingent shocks in a separate apparatus (TNC). For animals trained in the afternoon, the retention functions observed were monotonic in all cases (i.e., no Kamin effects were obtained). Experiment 2 compared groups that received avoidance training and retention testing in the same apparatus with groups that were trained and tested in a different apparatus. A Kamin effect was observed for the former groups but not for the latter.
\end{abstract}

Nonmonotonic retention functions in animals (i.e., U-shaped "Kamin" and multiphasic effects) have been widely observed (cf. Anisman, 1975; Holloway \& Wansley, 1973). Two major hypotheses have been proposed to account for such retention functions. One emphasizes certain associative mechanisms, such as deficits in memory retrieval (e.g., Bryan \& Spear, 1976) or state-dependent processes (Holloway \& Wansley, 1973). The second hypothesis attributes such retention functions to the organism's level of motivation and/or activity resulting from experience with an aversive stimulus (e.g., shock) during training (e.g., Anisman, 1975). Both of these positions have received considerable empirical support (cf. Anisman, 1975; Bryan \& Spear, 1976). Thus, one purpose of the present experiments was to provide additional information regarding the efficacy of these two hypotheses.

Recently, Elson, Seybert, and Ghiselli (1977) and Seybert, Vandenberg, Harvey, Budd, and McClanahan (1979) have reported findings that support the hypothesis that a similar (or the same) underlying mechanism is responsible for both Kamin and multiphasic retention functions and that, in fact, both functions represent the same retention process sampled at different retention intervals. Further support for such a suggestion would be provided if it could be demonstrated that both are influenced or determined by a significant variable in the same way.

Time of training may be such a variable. Elson et al. (1977) observed multiphasic functions for groups of animals trained in the morning (9:00 a.m.), but not for

Requests for reprints should be sent to J. A. Seybert, whose current address is Office of Institutional Research, Johnson County Community College, Overland Park, Kansas 66210. groups trained in the afternoon (4:00 p.m.). Thus, a second purpose of the present experiments was to determine the effect, if any, of time of training on the Kamin effect.

\section{EXPERIMENT 1}

This experiment examined the retention of an active avoidance response at three testing-training intervals (TTIs: .05, 1, and $24 \mathrm{~h}$ ). In addition, retention performance of two control conditions was also measured: a noncontingent group (labeled "pseudononcontingent," PNC), which received only footshock in the shuttlebox without an opportunity to escape or avoid during training, and a "truly noncontingent" (TNC) group, which received inescapable, unavoidable footshocks "off-line" during training. Finally, separate groups of animals in each of these conditions were trained either in the morning or in the afternoon.

\section{Method}

Subjects. The subjects were 144 male albino rats of the Sprague-Dawley strain obtained from the Holtzman Company. All subjects were approximately 125 days old when the experiment began, received ad tib food, and were individually housed. A 12-h light (7:00 a.m.-7:00 p.m.) and 12-h dark (7:00 p.m.7:00 a.m.) cycle was imposed for a 3-week period prior to training and for the duration of the experiment for all subjects. The animals were assigned randomly to 1 of 18 independent groups $(\mathrm{N}=8)$.

Apparatus. The apparatus consisted of two units. A one-way shuttlebox, $66 \times 23 \times 26 \mathrm{~cm}$ high, was employed for all subjects. The gray, wooden cubicle had a grid floor of $.6 \mathrm{~cm}$ parallel stainless steel bars separated by $1.5 \mathrm{~cm}$, had a hinged Plexiglas top, and contained two compartments, a startbox and a goalbox. The compartments were divided by a guillotine door that was operated by the experimenter. Shock onset and offset were controlled by a microswitch located at the startbox door and a photobeam $7 \mathrm{~cm}$ into the goalbox. A Model 113-33 Lehigh 
Valley shock generator was programmed to deliver a $1.0-\mathrm{mA}$ shock to the startbox grid $5 \mathrm{sec}$ after the door was raised. The shock was terminated when the subject crossed into the goalbox and interrupted the photobeam.

The second unit was employed for subjects in the TNC shock condition. The clear Plexiglas cubicle was $30 \times 15 \times 9 \mathrm{~cm}$ wide, had a grid floor of $.5-\mathrm{cm}$ parallel stainless steel bars separated by $1.0 \mathrm{~cm}$, and had a hinged Plexiglas top. The same shock generator used in the escape-avoidance box delivered the same amount of shock through the grid floor of the cubicle. Onset and termination of shock were controlled by a manual switch on the shock generator.

Procedure. The experiment consisted of a training phase and a testing phase. The 18 groups constituted the cells of a $3 \times 3 \times 2$ factorial design with training condition [active avoidance (AA), PNC, and TNC footshock], TTI (.05, 1, $24 \mathrm{~h})$, and time of training (morning and afternoon) serving as the factors.

During training, the AA condition consisted of the following procedure: Each subject was placed in the startbox facing away from the door. After $10 \mathrm{sec}$, the guillotine door was raised, and $5 \mathrm{sec}$ later, the grid floor was charged. To avoid the shock, the animal had to leave the startbox within $5 \mathrm{sec}$ after the door was raised. After an escape or avoidance, the animal remained in the goalbox for $15 \mathrm{sec}$ and was then returned to the startbox for the next trial. This procedure continued until each subject met a criterion of three successive avoidance responses.

Each subject in the AA conditions had two yoked counterparts, each of which received the same number and duration of shocks as the AA subjects. One such subject received shock in the startbox of the escape-avoidance chamber (PNC), and a second received shock in an off-line Plexiglas cubicle (TNC). All "morning" subjects were trained between 8:00 and 10:00 a.m., and all "afternoon" subjects were trained between 3:00 and 5:00 p.m. At $.05,1$, or $24 \mathrm{~h}$ following the training phase, the rats from each training condition were individually tested in the escape-avoidance apparatus. The testing phase for all subjects consisted of AA trials identical to the procedure used for AA subjects during training. The same avoidance criterion (i.e., three successive avoidances) was used during both training and testing. The dependent variables of interest were trials to criterion, the number of failures to avoid, and the percent of avoidances during testing.

\section{Results and Discussion}

Training. Analysis of the training data (trials to criterion) for both morning and afternoon AA groups indicated that neither the main effects of time of training and TTI nor their interaction was significant $(\mathrm{Fs}<1)$.

Testing. The data from the testing phase for all three dependent variables are depicted in Figures 1 and 2. As can be seen in Figure 1, for animals trained in the morning, both the AA and PNC conditions evidenced nonmonotonic (approximately U-shaped) retention functions for each of the dependent variables, whereas retention for the TNC condition was essentially monotonic. As can be seen in Figure 2, however, the pattern of results was quite different for afternoon-trained animals. Specifically, it appears that for all three dependent variables, retention for the AA groups was only very slightly U-shaped, decreased monotonically for the PNC groups, and was constant across TTI for the TNC groups (and considerably below the AA and PNC conditions).

The data were evaluated by 2 (time of training) by 3 (TTI) by 3 (training condition) factorial analyses of variance conducted for each dependent variable. The time of training main effect was not significant for any of the three dependent variables (all Fs $<1$ ). The TTI
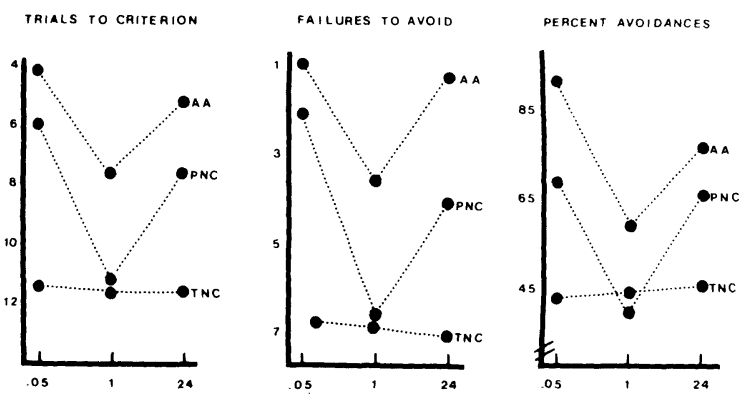

RETENTION INTERVAL (TTI-HOURS)

Figure 1. Mean trials to criterion, failures to avoid, and percent avoidances as a function of training condition and TTI for subjects trained in the morning (Experiment 1).
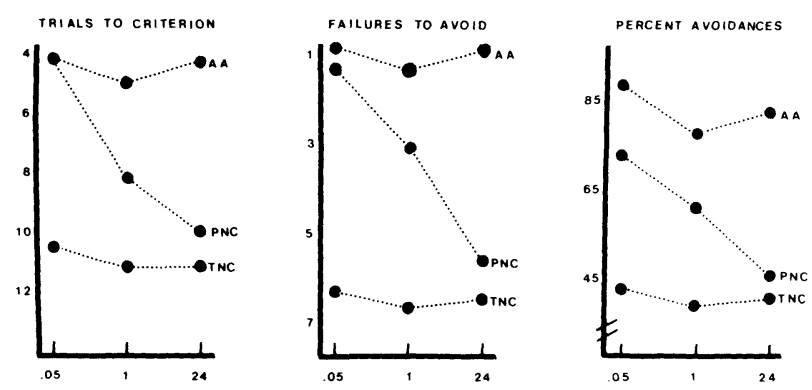

RETENTION INTERVAL (TTI-HOURS)

Figure 2. Mean trials to criterion, failures to avoid, and percent avoidances as a function of training condition and TTI for subjects trained in the afternoon (Experiment 1).

main effect was significant for all three dependent variables $[\mathrm{Fs}(2,111)=7.86,8.46$, and 11.60 , ps $<.005$, for trials to criterion, percent avoidances, and failures to avoid, respectively]. The training condition main effect was also significant for all three dependent variables $[\mathrm{Fs}(2,111)=36.26,47.82$, and 57.17 , ps $<.001$, respectively]. The Time of Training by Training Condition interaction was not significant for any of the dependent variables $(\mathrm{Fs}<1)$. The Time of Training by TTI interaction was significant for the percent avoidances dependent variable $[F(2,111)=3.95, p<.05]$, but it was not significant for trials to criterion and failures to avoid $[\operatorname{Fs}(2,111)=2.46$ and 2.51 , ps $>.05$, respectively $]$. The Training Condition by TTI interaction was significant for failures to avoid $[F(4,111)=5.79, p<.001]$, and percent avoidances $[\mathrm{F}(4,111)=5.30, \mathrm{p}<.001]$, but only marginally so for trials to criterion $[F(4,111)=$ $2.18, p=.07]$. The Time of Training by TTI by Training Condition interaction was significant for all three dependent variables $[F s(4,111)=3.38,2.95$, and 4.16 , ps $<.025$, for trials to criterion, failures to avoid, and percent avoidances, respectively].

In order to further evaluate the present data, individual analyses of variance were performed on each of the six training condition groups as a function of TTI for all three dependent variables. For subjects trained in 
the morning, no significant effect of TTI was apparent in the TNC condition for any of the dependent variables ( $F<1$ in all cases), but a significant TTI effect was obtained in the AA groups $[F s(2,21)=5.31,8.44$, 15.02 , ps $<.01$, for trials to criterion, failures to avoid, and percent avoidances, respectively]. The effect of TTI was also significant for the PNC groups $[\mathrm{Fs}(2,21)=$ $7.48,8.88,8.16$, ps $<.005$, for trials to criterion, percent avoidances, and failures to avoid, respectively]. Subsequent trend analyses indicated that the TTI effect in both AA and PNC conditions was primarily quadratic in nature $[\mathrm{Fs}(1,21)=9.42$ and 13.24 , ps $<.005$, for trials to criterion; $\operatorname{Fs}(1,21)=20.25$ and 32.22 , ps $<.005$, for percent avoidances; and $\operatorname{Fs}(1,21)=15.60$ and 13.07 , ps $<.005$, for failures to avoid, respectively].

Identical analyses on the data from the afternoontrained animals indicated that results for the TNC groups were very similar to those for morning-trained animals. Namely, the TTI effect was not significant for any of the dependent variables (all $F_{s}<1$ ). The pattern of results for the afternoon AA groups was very different from that obtained for morning-trained subjects, however. The TTI effect was not significant for trials to criterion $(\mathrm{F}<1)$, percent avoidances $(\mathrm{F}<1)$, or failures to avoid $[F(2,21)=2.86, p>.05]$.

Finally, for the afternoon PNC subjects, the TTI effect was also significant $[\mathrm{Fs}(2,21)=7.27,15.71$, and $9.67, \mathrm{ps}<.001$, respectively, for the trials to criterion, failures to avoid, and percent avoidances dependent variables]. As can be seen in Figure 2, retention performance declined nonmonotonically for the PNC animals across retention intervals. This observation is reflected in the trend analyses, which indicated significant linear trend components for all three dependent variables $[\mathrm{Fs}(1,21)=14.27,19.17$, and 31.10 , ps $<.001$, for trials to criterion, failures to avoid, and percent avoidances, respectively]. The reasons for the relative retention loss across TTI for the PNC groups as compared with the AA and TNC groups are not clear.

In addition to the expected finding of a Kamin effect for the AA "morning" groups, the observation of such an effect in the PNC "morning" groups confirms those reported in previous investigations of retention following experience with "noncontingent" shock (e.g., de Toledo \& Black, 1970). Similarly, in agreement with the present TNC findings, de Toledo and Black reported a failure to observe a Kamin effect in groups preshocked in one apparatus and tested for avoidance responding in another. The literature, then, is in clear agreement with the present findings that, in the absence of overt response training, on-line preshock (PNC) will produce a Kamin effect in a subsequent avoidance retention test, whereas off-line preshock (TNC) fails to produce such an effect. Thus, while the presence or absence of a discrete instrumental response is not necessary for the occurrence of a Kamin effect, a certain amount of similarity between the stimuli present during training and those present during testing does seem to be a necessary condition for the occurrence of such an effect. These find- ings strongly indicate that the reinstatement (retrieval) of an association involving a stimulus or set of stimuli present during training is absolutely necessary for the occurrence of the Kamin effect.

\section{EXPERIMENT 2}

One important set of circumstances present for both AA and PNC "morning" groups in Experiment 1 was placement in the gray startbox, followed by exposure to shock. It is probable, then, that this S-S association (between apparatus and shock) was formed and was extremely salient for animals in both conditions. Also, it is possible that retention of this association is reflected in the Kamin effects evidenced by these groups. Thus, animals trained to avoid and tested in the same apparatus would be expected to evidence a Kamin effect, but animals trained to avoid in one apparatus and tested in another would not.

\section{Method}

Subjects. The subjects were 36 rats exactly like those used in Experiment 1, and they were housed and fed under exactly the same conditions. They were randomly assigned to one of six groups prior to the start of the experiment $(N=6 /$ group).

Apparatus. Two separate apparatus units were used in this experiment. The first was the gray wooden shuttlebox used in Experiment 1. The second unit consisted of a $60 \times 15 \times 9 \mathrm{~cm}$ one-way Plexiglas shuttlebox. This unit contained a grid floor of $.5-\mathrm{cm}$ parallel stainless steel bars separated by $1.0 \mathrm{~cm}$ and a guillotine door that divided the apparatus into two $30-\mathrm{cm}$ tong compartments (startbox and goalbox). Shock onset and offset were controlled by a microswitch at the guillotine door and a photobeam $7 \mathrm{~cm}$ inside the goalbox. Shock was delivered to both units as in Experiment 1.

Procedure. The experiment consisted of a training phase and a testing phase. The six groups constituted the cells of a 2 by 3 factorial design with training condition (test apparatus same as training, "S," or different from training, "D") and retention interval $(.05,1,25 \mathrm{~h})$ serving as the factors. For the animals in the $\mathrm{S}$ conditions, the procedure was identical to that for the AA groups used in Experiment 1 for both training and testing. The animals in the $D$ conditions were treated identically to those in the $S$ conditions, with the exception that their avoidance training took place in the smaller Plexiglas apparatus. In all other respects, the animals in both conditions experienced the same procedures as the AA (morning) animals in the first experiment. All subjects in the present experiment were trained between $8: 00$ and 10:00 a.m.

\section{Results and Discussion}

Training. Analysis of the training data indicated no significant main effects or interactions (all Fs $<1$ ).

Testing. The data from the testing phase for all three dependent variables are depicted in Figure 3. Generally, the retention functions for the $S$ groups appeared to be approximately U-shaped, whereas those for the D groups increased monotonically as a function of TTI. These observations were confirmed by the statistical analyses conducted on the data. The 2 (training condition) by 3 (TTI) factorial analyses conducted on the three dependent variables yielded significant main effects for training condition $\left[F_{s}(1,30)=47.83,59.93\right.$, and 71.60 , ps $<.001$, for trials to criterion, failures to avoid, and 

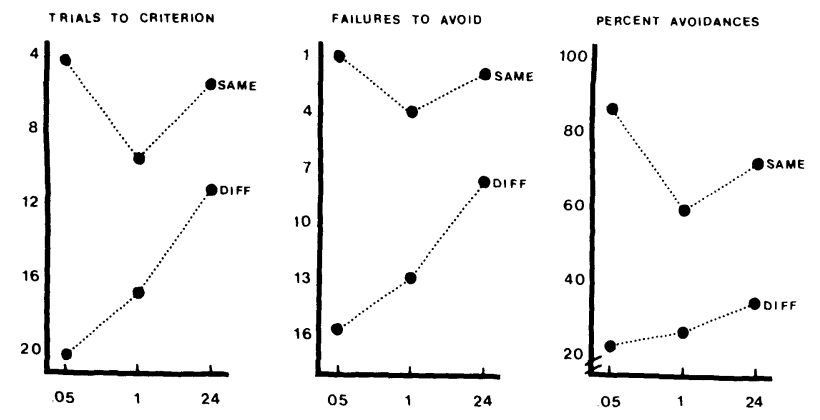

RETENTION INTERVAL (TTI-HOURS)

Figure 3. Mean trials to criterion, failures to avoid, and percent avoidances as a function of training condition and TTI in Experiment 2.

percent avoidances, respectively]. In addition, the TTI main effect was significant for trials to criterion $[F(2,30)=4.24, \quad p<.05]$ and failures to avoid $[F(2,30)=3.35, p<.05]$, but not for percept avoidances $[F(2,30)=2.00, p>.10]$. Finally, the Training Condition by TTI interaction was significant in all cases $[F s(2,30)=5.20,3.99$, and 3.43 , ps $<.05$, for trials to criterion, failures to avoid, and percent avoidances, respectively].

The data were examined further using one-way analyses of variance and trend analyses on the different training conditions. The analyses of variance revealed significant effects of TTI in the S groups for trials to criterion $[F(2,15)=33.82, p<.001]$ and failures to avoid $[F(2,15)=5.12, p<.05]$, but not for percent avoidances $[F(2,15)=2.07, p>.05]$. The results for the D groups indicated significant effects of TTI for trials to criterion $[\mathrm{F}(2,15)=4.11, \mathrm{p}<.05]$ and failures to avoid $[\mathrm{F}(2,15)=3.71, \mathrm{p}=.05]$, but not for percent avoidances $[F(2,15)=2.00, p>.05]$. The trend analyses indicated that the TTI effect was primarily quadratic in nature for the $S$ groups $[\mathrm{Fs}(1,15)=51.58$ and 9.16 , ps $<.01$, for trials to criterion and failures to avoid, respectively] and that the effect of TTI was linear for the $\mathrm{D}$ groups $[\mathrm{Fs}(1,15)=8.08$ and 6.25 , ps $<.025$, for the same two dependent variables].

The important finding in the present experiment is the observation of a Kamin effect in the $S$ groups, which received training and testing in the same apparatus, and the absence of any nonmonotonic retention function in the D groups, which were trained in one apparatus and then tested in a second. Since these groups learned identical responses but produced distinctly different retention functions, we must conclude that some factor other than retention of the discrete instrumental response itself is responsible for the Kamin effect observed in the $S$ groups. Since we have previously demonstrated that retention of a learned association is what is reflected in nonmonotonic retention phenomena (both Kamin and multiphasic effectssee Experiment 1 reported here; Elson et al., 1977; Seybert et al., 1979) and the present results indicate that the avoidance response is not involved in that association, other important features of the training environment must make up that association. Thus, we believe that the Kamin effects observed in this experiment reflect retention of an association between apparatus cues and shock, rather than an association involving the instrumental avoidance response itself.

\section{GENERAL DISCUSSION}

We believe that the results of the experiments reported here are important for several reasons. First, a Kamin effect was observed in morning-trained subjects but not in afternoontrained subjects (Experiment 1). These findings are strikingly similar to those from experiments involving the same manipulations in the multiphasic retention paradigm (Elson et al., 1977). Taken together, then, the data reported by Elson et al. and those of Experiment 1 here provide support for the suggestion that Kamin and multiphasic retention phenomena reflect the same underlying mechanism or process sampled at different TTI.

Second, the data from morning-trained animals in Experiment 1 , which indicated very different retention functions for the AA and PNC groups compared with the TNC groups, provide confirmation of the associative/memory hypothesis of nonmonotonic retention. Since all three conditions received identical exposure to shock, in terms of amount, frequency, and duration, the nonassociative hypotheses would have predicted identical retention functions.

Finally, the data from Experiment 2 indicate that retrieval of an S-S association is responsible for nonmonotonic retention effects. Such associations might also be thought of as learned motives (like incentive in appetitive situations, or fear in the present context) or S-S* expectancies, as Bolles (1972) has proposed. We have suggested previously (Seybert et al., 1979) that nonmonotonic retention functions, like the Kamin effects reported here, represent differential retrieval of these types of expectancies or learned motives, rather than of an association involving the instrumental response. The present data, we believe, provide further support for such a contention.

\section{REFERENCES}

Anisman, H. Time dependent variations in aversively motivated behaviors: Nonassociative effects of cholinergic and catecholaminergic activity. Psychological Review, 1975, 82, 359-385.

Bolles, R. C. Reinforcement, expectancy, and learning. Psychological Review, 1972, 79, 394-409.

Bryan, R. C., \& SPEAR, N. E. Forgetting of a discrimination after intervals of intermediate length: The Kamin effect with choice behavior. Journal of Experimental Psychology: Animal Behavior Processes, 1976, 2, 221-234.

DE TOledo, L., \& BlACK, A. H. Retention of aversively motivated responses in rats. Journal of Comparative and Physiological Psychology, 1970, 71, 276-282.

Elson, I. J., Seybert, J. A., \& Ghiselli, W. B. Retention of aversively motivated behavior: Effects of time of training and associative versus nonassociative processes. Behavioral Biology, $1977,20,337-353$.

Holloway, F., \& Wansley, R. Multiple retention deficits at periodic intervals after active and passive avoidance learning. Behavioral Biology, 1973, 9, 1-14.

Seybert, J. A., Vandenberg, G. L., Harvey, R. J., Budd, J. R., \& McClanahan, L. G. Retention of appetitive instrumental behavior: The Kamin effect. Behavioral and Neural Biology, 1979, 26, 266-286. 